Check for updates

Cite this: Phys. Chem. Chem. Phys., 2021, 23, 7758

Received 9th August 2020,

Accepted 18th September 2020

DOI: $10.1039 / \mathrm{d} 0 \mathrm{cp} 04221 \mathrm{~h}$

rsc.li/pccp

\section{Full-dimensional potential energy surface for acetylacetone and tunneling splittings $\dagger$}

\author{
Chen Qu, (D) ${ }^{a}$ Riccardo Conte, (D) *b Paul L. Houston (D) *cd and Joel M. Bowman (D) *e
}

We present a full-dimensional potential energy surface for acetylacetone (AcAc) using full and fragmented permutationally invariant polynomial approaches. Previously reported MP2/aVTZ energies and gradients are augmented by additional calculations at this level of theory for the fits. Numerous stationary points are reported as are the usual metrics to assess the precision of the fit. The electronic barrier height for the $\mathrm{H}$-atom transfer is roughly $2.2 \mathrm{kcal} \mathrm{mol}^{-1}$. Diffusion Monte Carlo (DMC) calculations are used to calculate the ground state wavefunction and zero-point energy of acetylacetone. These together with fixed-node DMC calculations for the first excited-state provide the predicted tunneling splitting due to the barrier to $\mathrm{H}$-transfer separating two equivalent wells. Simpler $1 \mathrm{~d}$ calculations of this splitting are also reported for varying barrier heights including the CCSD(T) barrier height of $3.2 \mathrm{kcal} \mathrm{mol}^{-1}$. Based on those results the DMC splitting of $160 \mathrm{~cm}^{-1}$ with a statistical uncertainty of roughly $21 \mathrm{~cm}^{-1}$, calculated using the MP2-based PES, is estimated to decrease to $100 \mathrm{~cm}^{-1}$ for a barrier of $3.2 \mathrm{kcal} \mathrm{mol}^{-1}$. The fragmented surface is shown to be fast to evaluate.

\section{Introduction}

It is a truism that potential energy surfaces (PESs) play a central role in all of computational chemistry. There has been breathtaking progress in the past 15 years in developing so-called nonparametric, machine learning approaches to fit large data sets of electronic energies. These include permutationally invariant polynomials (PIPs), Gaussian process regression (GPR), neural networks (NN), combinations of all three as well as variants of the GPR and NN approaches. Numerous reviews have appeared that cover much of this field and we refer the reader to a handful that provide a good overview. ${ }^{2-9}$ These methods have steep scaling with respect to the number of atoms in the molecule or cluster and the size of the data set. (By far the steepest scaling is with respect to the electronic energy calculation.) Several comparative studies of these methods have recently appeared. ${ }^{10-13}$

These methods all have in common that they do not rely on a model for representing potentials, e.g., Lennard-Jones, LEPS,

\footnotetext{
${ }^{a}$ Department of Chemistry \& Biochemistry, University of Maryland, College Park, Maryland 20742, USA

${ }^{b}$ Dipartimento di Chimica, Università Degli Studi di Milano, via Golgi 19, 20133 Milano, Italy. E-mail: riccardo.conte1@unimi.it

${ }^{c}$ Department of Chemistry and Chemical Biology, Cornell University, Ithaca, New York 14853, USA. E-mail: plh2@cornell.edu

${ }^{d}$ Department of Chemistry and Biochemistry, Georgia Institute of Technology, Atlanta, Georgia 30332, USA

${ }^{e}$ Cherry L. Emerson Center for Scientific Computations and Department of Chemistry, Atlanta, Georgia 30322, USA. E-mail: jmbowma@emory.edu

$\dagger$ Electronic supplementary information (ESI) available. See DOI: 10.1039/d0cp04221 h
}

exp/6, force-fields, etc. In this sense they are all non-parametric in the language of machine-learning (ML). Also, they use a universal set of inputs, also known as descriptors, for the fitting. In the PIP approach the input is all Morse variables, which are transformed internuclear distances. In the PIP-NN ${ }^{6,14}$ and PIP-GPR ${ }^{11}$ the input is a limited number of PIPs. This is distinct from earlier approaches using models, where the variables are molecule-specific. So, based on these aspects, all the methods mentioned above (not a comprehensive list) are machine-learning methods. However, there is a fundamental difference in the way the data are used in these methods. In PIP and NN the parameters contained in those approaches are optimized, generally using a least squares minimization criterion. In PIP, where parameters are linear, the optimization is performed by means of linear least-squares. In NN the fitting parameters are non-linear and so the least squares optimization is non-linear. The number of parameters in both approaches can be thousands or even tens of thousands, depending on the dimensionality of the space. As an aside, it is worth noting that if Morse variables are used as the inputs for PIP or PIP-NN, then the range parameter(s) of the variables could be treated as additional non-linear parameters. The number of such parameters is very small, however, compared to the thousands of parameters just mentioned. In any case, in these methods the data are used in the optimizations but not for prediction. By contrast, other machine-learning methods use the data explicitly for prediction. GPR is a prominent example of this method. All these methods have the same goals, which are a precise representation of the known data (GPR can reproduce 
the data exactly but, since this generally leads to a singular matrix for prediction, "noise" is added to the data) and smooth and accurate predictions. It is of course of interest to compare the performance of these different ML approaches.

Based now on numerous applications using these methods, some fairly general comments can be made about them. A strength of the GPR approach is that it does produce a quality PES from a relatively small amount of data., ${ }^{3,15}$ On the other hand this approach becomes computationally very demanding for datasets with more than $10^{4}$ energies. By contrast, PIP/ FI-NN PESs with 4-7 atoms typically use more than $10^{4}$ energies. ${ }^{6,8}$ For example, the recent PIP-NN and atom-based HD-NN PESs for $\mathrm{H}+\mathrm{CH}_{3} \mathrm{OH}$ were fit to 75000 electronic energies. ${ }^{13}$ The PIP approach developed in our group spans both these limits, i.e., it can easily fit $c a .10^{5}$ energies (and/or gradients) and it also provides a faithful fit with very few configurations. This was explicitly the focus of a recent paper assessing the performance, i.e., speed and accuracy, of the GPR and PIP approach to PES fitting for four case studies, $\mathrm{H}_{3} \mathrm{O}^{+}$, $\mathrm{OCHCO}^{+}, \mathrm{H}_{2} \mathrm{CO}$ (including cis- and trans- $\mathrm{HCOH}$ isomers) and 10 atom formic acid dimer. ${ }^{11}$ That paper, which also extended the GPR method to use PIP inputs, examined the fidelity of GPR and PIP fits for these four molecules with respect to size of the training set of energies as well as the time for fitting and evaluation. One example is the PES for 5-atom $\mathrm{OCHCO}^{+}$. The original PIP PES was constructed with just 8613 CCSD(T)-F12/aVTZ energies. ${ }^{16}$ GPR, PIP-GPR and PIP fits were done using between 520 and 2600 scattered energies. All methods fit the training data with about equal precision; however, the GPR precision was significantly better for the remaining test data. The PIP fits were roughly 60 times faster to evaluate than the GPR ones. Both fits, using 1560 training data, gave harmonic frequencies at the minimum and proton transfer saddle point with maximum differences from direct $a b$ initio results of no more than $4 \mathrm{~cm}^{-1}$.

Finally, on the matter of data size and PES fitting, we note that our PIP software was recently extended to fit gradients as well as energies. ${ }^{17}$ With this approach a PIP PES for $\mathrm{CH}_{4}$ was obtained at the DFT level of theory with just 100 configurations. In a more recent application, a PIP PES for 12-atom $N$-methyl acetamide was reported using a dataset of 244459 energies and gradients. ${ }^{18}$ An even larger applicaton was to 15 -atom tropolone ${ }^{19}$ with a data size of 311328 .

To summarize this short review of these ML methods, all can achieve high precision, depending on the application. In our opinion it is not particularly meaningful to focus on a root-mean-square (RMS) error difference of even tens of $\mathrm{cm}^{-1}$ between methods, except in cases where the electronic energies being fit are themselves within or less that level of accuracy. Perhaps speed of evaluation of the PES and for many applications the gradient of the energy are more relevant.

As for applications, PESs for reaction dynamics are perhaps the most demanding. By definition, reactions involve large amplitude motions and so local methods, e.g., harmonic or perturbative extensions of harmonic theory, are totally inadequate. Second, bond breaking is demanding on electronic structure methods. Third, many reactions of interest involve $\mathrm{H}$-atom motion and this can require a quantum or semi-classical treatment of the dynamics. Thus, PESs for reactive systems are a major challenge as they must describe large, indeed very large amplitude motion. The above ML methods have been employed over the past 10 or so years to develop high-dimensional PESs for reactive systems. ${ }^{8,9,12,13,20-23}$ Some time ago $\mathrm{Fu}$ et al. reported a PIP PES for the 7-atom $\mathrm{O}\left({ }^{3} \mathrm{P}\right)+\mathrm{C}_{2} \mathrm{H}_{4}$ reaction (which included spin-orbit coupling to the singlet PES). This PES was used in quasiclassical trajectory calculations which yielded excellent agreement with experiment for the branching ratio of numerous products. ${ }^{23}$ Recently, Li et al. reported PIP-NN and HD-NN PESs for several chemical reactions. ${ }^{12,13}$ The most recent example is the 7-atom $\mathrm{H}+\mathrm{CH}_{3} \mathrm{OH}$ reaction. Although these are both neural network methods, they are different in the way the potential is represented. In the HD-NN one the energy is represented as a sum of atomic energies and there is a neural network for each atom (identical atoms share the same set of NN parameters). So in this example there are 7 NNs. For the PIP-NN ${ }^{6}$ approaches there is one NN and the input consists of a limited number of PIPs. ${ }^{6}$ (Also, see the related fundamental invariant-NN (FI-NN) approach. ${ }^{8,14}$ ) The authors noted that the PIP-NN PES is faster to evaluate than the HD-NN one. This is as expected since the number of parameters in the $\mathrm{NN}$ in each approach is about the same. However, the strength of the atom-based HD-NN approach is that it scales linearly with the number of atoms and so it is feasible to apply to large systems, ideally of the same atom type. The strength of the PIP and the PIP-NN approaches lies in the speed and high precision for molecular systems of around 10 atoms. The speed of the PIP-NN approach decreases significantly with the number of atoms because the number of PIP inputs grows non-linearly with the number of atoms. ${ }^{24,25}$ Recently, Zhang and co-workers have extended the techniques in the FI-NN approach to ten atoms ${ }^{26}$ however, without yet reporting a 10-atom PES.

Among reactive systems, perhaps the simplest ones are isomerization reactions and, among these, symmetric double well isomerizations are the most studied. Of these malonaldehyde is perhaps the most studied both experimentally and theoretically. In 2008 we reported a PIP PES for the 9-atom malonaldehyde ${ }^{27}$ This was a precise fit to highly-accurate "focal point" electronic energies. This PES was subsequently used in various full-dimensional calculations of the tunneling splittings for $\mathrm{H}$ and D-transfer and finally agreement (to within less than $10 \%$ ) with experiment was obtained for both. A much more recent isomerization example is 15-atom tropolone, for which we reported a PIP PES. ${ }^{19}$ For this PES, owing to the very high computational cost of $\operatorname{CCSD}(\mathrm{T})$ calculations, DFT ones were done for energies and gradients. Thus, this PES, without further modification, will not yield quantitative tunneling splittings.

Clearly, there is major motivation to extend all ML methods to large molecules of interest in chemistry and specifically reaction dynamics. We recently described a strategy to do this for the PIP approach. ${ }^{18,28}$ The key points derive from the fact the fitting basis is made of permutationally invariant polynomials of Morse variables. For an $n$-atom molecule there are $n(n-1) / 2$ variables and so this quadratic dependence on $n$ is not favorable for large molecules. A second issue is the non-linear 
growth in the number of terms in the basis, for a fixed total polynomial order. For example, for 12-atom $N$-methyl acetamide there are 66 Morse variables and with a highly reduced permutational symmetry 33111111 , which accounts for the symmetry of the three $\mathrm{H}$ atom of the two distant methyl groups, the basis contains 8040 terms (and unknown linear coefficients) at maximum polynomial order of 3, but grows to 95965 at polynomial order of 4 . (Increasing the permutational symmetry reduces the number of terms dramatically; ${ }^{2}$ however, with a much increased cost to obtain them and often also to evaluate each basis function.) The strategy mentioned above is briefly reviewed next.

\section{Short review of fragmented PIP theory}

To begin, recall that in the PIP approach the potential is given by the compact expression:

$$
V=\sum_{i=1}^{n_{\mathrm{p}}} c_{i} p_{i},
$$

where $c_{i}$ are coefficients, $p_{i}$ (the basis set functions) are PIPs, and $n_{\mathrm{p}}$ is the total number of polynomials for a given maximum polynomial order. The $p_{i}$ are generally functions of Morse variables, which themselves are functions of the inter-atomic distances, $r_{\alpha, \beta}$ (by the usual exponential relationship $\exp \left(-r_{\alpha, \beta} / \lambda\right)$, where $\lambda$ is commonly chosen to be equal to 2 Bohr). The Morse variables are denoted by $x_{l}$. The linear coefficients are obtained using standard least squares fits to large data sets of electronic energies (and possibly also gradients) at "scattered" geometries.

In the usual approach, a serious computational issue arises when the basis set is too large to be practically useful, either because the number of coefficients is so large that the least squares optimization becomes problematic or because calculating the PIPs becomes expensive. The size of the basis depends in a complicated and non-linear way on the maximum polynomial order, the number of Morse variables, and the order of the symmetric group. ${ }^{25}$ This growth in the size of the PIP basis was the origin of for the estimate of a 10-atom limit for the method. ${ }^{25}$

However, as noted above, the fragmented basis approach is an effective way to deal with this apparent 10-atom limit. By fragmenting a molecule into groups of smaller moieties the basis for each moiety can be calculated rapidly and then combined with those of other fragments to provide a compact and hopefully still precise representation of the PES. ${ }^{28}$ Consider a simple example of a 5-atom molecule with atoms labeled as 1-5 and a scheme in which the molecule is fragmented into three fragments, say $\{1,2,3\},\{2,3,4\},\{3,4,5\}$. In this 3 -fragment scheme the potential is given compactly by

$$
V=\sum_{i} c_{i} p_{i}\left(\boldsymbol{x}_{1}, \boldsymbol{m}_{1}\right)+\sum_{j} c_{j}^{\prime} p_{j}^{\prime}\left(\boldsymbol{x}_{2}, \boldsymbol{m}_{2}\right)+\sum_{k} c_{k}^{\prime \prime} p_{k}^{\prime \prime}\left(\boldsymbol{x}_{3}, \boldsymbol{m}_{3}\right),
$$

where $\{p\},\left\{p^{\prime}\right\}$, and $\left\{p^{\prime \prime}\right\}$ are PIP bases for the $n$th fragment, $n=1,2,3,\{c\},\left\{c^{\prime}\right\},\left\{c^{\prime \prime}\right\}$ are the corresponding linear coefficients, $\boldsymbol{x}_{n}$ represent the set of corresponding Morse variables, and $\boldsymbol{m}_{n}$ indicate a set of monomials built from the Morse variables. Morse variables between atoms 1 and 4, atoms 1 and 5, and atoms 2 and 5 are assumed to be zero and hence not included in the fragmented bases.

In this example, and in general, there are some Morse variables in common among the fragments, and thus there are some redundant basis functions in this expression in terms of common Morse variables. These issues were pointed out previously; ${ }^{18,28}$ however, they were not serious numerically, because the linear least squares method used is able to deal with a modest number of identical basis functions. Nevertheless, there is motivation to eliminate these redundant basis functions and thereby reduce the size of the basis. We do note the redundant-term issue is similar to one that two of us identified earlier for developing PIP representations of interaction potentials that should rigorously vanish in asymptotic regions where there is no inter-fragment interaction. In that case the issue was basis functions involving Morse variables of fragments that do not go to zero at large internuclear distances where there is no inter-fragment interaction. An effective pruning procedure was then employed to eliminate such basis functions and applied to several systems. ${ }^{29-31}$

It is worth recalling the key point that motivated the fragment approach. The PIP basis can be generated by starting with "seed" monomials ${ }^{24}$ given by eqn (3)

$$
x_{1}^{n_{1}} x_{2}^{n_{2}} \ldots x_{l}^{n_{l}}, \quad l=n(n-1) / 2,
$$

where the Morse variables are indexed by an integer. Clearly for large molecules, many internuclear distances are large and thus the corresponding Morse variables are approximately zero, and so basis functions containing these variables are also zero. One way to take advantage of this is to simply prune the full basis and then remove those basis polynomials with very small Morse variables. However, this requires obtaining the full fitting basis first before pruning, and this could already be prohibitive. So a fragmented approach was proposed in which the basis is a union of PIPs for fragments of the molecule. In order to maintain permutational invariance for the final basis set, atoms that are assigned to permute with one another must appear together whenever they appear in any of the fragments.

As noted already this approach was applied successfully to cis and tran-NMA (including the saddle point(s)) between them. Very recently it was applied to 15 -atom tropolone, ${ }^{19}$ where a full PIP basis was also used.

Here we apply the fragmented PIP approach to obtain a PES 15-atom acetylacetone (AcAc). (We use the PES to obtain the $\mathrm{H}$ and D-tunneling splitting.) It is worth going over the history of AcAc PEs for the usual reasons and also since the molecule is an excellent example of the rapid pace of progress mentioned above. In 1997 Hinsen and Roux reported an empirical valence bond (EVB) potential (using input from a well-known force field and limited HF calculations). ${ }^{32,33}$ The barrier for the $\mathrm{H}$-atom transfer of roughly $9 \mathrm{kcal} \mathrm{mol}^{-1}$ is about a factor of three larger than the current best value from $\operatorname{CCSD}(\mathrm{T})$ calculations of 
$3.2 \mathrm{kcal} \mathrm{mol}^{-1} \cdot{ }^{34}$ (This large overshoot of an HF-based barrier is certainly in-line with many other calculations of barriers using HF theory.) This was followed in 2001 with a systematic study of the barrier height using DFT/B3LYP and MP2 theory and a variety of basis sets by Mavri and Grdadolnik. ${ }^{22}$ To quote from that paper "For description of the intramolecular degrees of freedom... one would need 39-dimensional hypersurface, which is impossible to construct..." Considering accuracy based on MP2 calculations and speed the authors went on to develop a $4 \mathrm{~d}$ EVB potential based on B3LYP/6-311+G(2d,2p) calculations with a classical barrier of around $2 \mathrm{kcal} \mathrm{mol}^{-1}$. In 2015 Meuwly and co-workers developed and employed a full-dimensional semi-empirical molecular-mechanics-proton transfer PES (with input from MP2 calculations) to calculate a classical IR spectrum and to compare with a new experimental spectrum. In 2020, Meuwly and co-workers using a novel Neural Network approach in the PhysNet software ${ }^{35}$ developed a full-dimensional PES for AcAc based on MP2/aVTZ energies and gradients. ${ }^{1}$ This PES was used in molecular dynamics calculations of the infrared spectrum. Thus, in 19 years a full dimensional PES for 15-atom AcAc has gone from "impossible" to a reality.

In this paper we apply the fragmented PIP approach to obtain a new PES for AcAc. Although a NN-based PES for AcAc has recently been reported, ${ }^{1}$ as noted above, we decided to augment the data set for that fit with additional MP2/aVTZ energies and gradients to obtain and investigate a new PES. The two approaches are very different and so it is worthwhile to report on this new PES, providing additional insight on accuracy and computational timing. We use the new fit in diffusion Monte Carlo calculations of the ground vibrational state wavefunction and zero-point energy and fixed-node DMC calculations of the first excited state in the $\mathrm{H}$ and D-atom transfer motion to obtain the tunneling splitting. Also, a simple 1d approach to obtain the tunneling splitting is also used, mainly to determine the effect of increasing the barrier height on the tunneling splitting.

Of course, AcAc is just one example of the rapid progress in the field. Already in 2005 permutationally invariant PESs for $\mathrm{CH}_{5}{ }^{+}$and $\mathrm{H}_{5} \mathrm{O}_{2}{ }^{+}$based on fitting tens of thousands "gold standard" CCSD(T)/aVTZ energies were reported. ${ }^{36,37}$ The six and seven atom molecules were already "large" by the standards in 2005 and in both cases the PESs dissociated smoothly to fragments, $\mathrm{CH}_{3}{ }^{+}+\mathrm{H}_{2}$ and $\mathrm{H}_{3} \mathrm{O}^{+}+\mathrm{H}_{2} \mathrm{O}$, respectively.

\section{Computational details}

\subsection{PIP bases}

PIP software that incorporates gradients into fitting was introduced recently and first applied in a demonstration to $\mathrm{CH}_{4}{ }^{17}$ and in the first application of the fragmentation approach to $N$-methyl acetamide. ${ }^{28,38}$ This software was refined for the fragmented approach to eliminate redundant basis functions ${ }^{39}$ and very recently applied to obtain a full-dimensional PES for tropolone. ${ }^{19}$
We investigated two bases for the fits to the present data set. The first, which we assumed would be the benchmark, was a full PIP basis of maximum polynomial order three. The atom numbering scheme employed is shown in the top panel of Fig. 1, which also gives the minimum energy structure of AcAc. The symmetry designation for this fit is $\{1,2,2,2,6,1,1\}$, meaning that the two oxygens (atoms 2 and 3) are treated as equivalent, as are the two carbons (atoms 4 and 5), the two end carbons (atoms 6 and 7), and the six hydrogens (atoms 8-13) on the terminal carbons. The remaining $\mathrm{H}$ atoms and the central carbon are treated as unique.

The second fit we examined was based on fragmentation of AcAc into four fragments and again using a maximum polynomial order of three. The details of this approach have been described previously. ${ }^{18,28,39}$ The permutational symmetries and atom numbers (using the scheme in the bottom panel of Fig. 1) are as follows: symmetry $\{1,1,1,1,1,1,1,1,1\}$ with atoms $\{1,2$, $3,4,5,6,10,11,12\}$; symmetry $\{3,1,1,1,1,1,1,1,1\}$ with atoms $\{13,14,15,1,2,3,4,5,10,11,12\}$; symmetry $\{3,1,1,1,1$, $1,1,1,1\}$ with atoms $\{7,8,9,1,2,3,4,5,6,10,11\}$; and symmetry $\{3,3,1,1\}$ with atoms $\{7,8,9,13,14,15,6,12\}$. We will refer to this fragmentation as $4-(9,11,11,8)$, where the numbers in parentheses represent the number of atoms in each fragment.

Properties of these basis sets are shown in Table 1 along with their performance. At this point we just note that while the fragmented basis has more terms it is faster to evaluate for both the potential and gradient. This may seem counter intuitive;
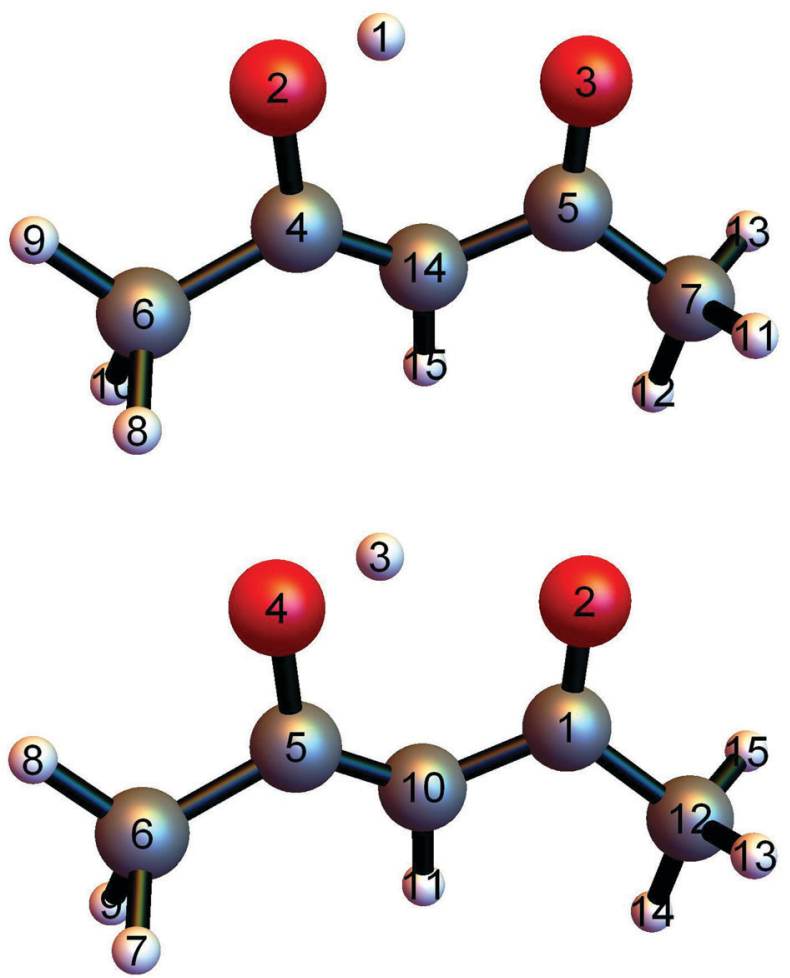

Fig. 1 (top) Numbering scheme used for the full-symmetry basis set. (bottom) Numbering scheme used for the $4-(9,11,11,8)$ basis set. 
Table 1 Basis sets investigated for AcAc. Weighted RMS error values (WRMSE) are in $\mathrm{cm}^{-1}$ and $\mathrm{cm}^{-1}$ per Bohr for potentials and gradient components, respectively. Times are in $\mathrm{s}$ for an average over 10 tests using a $2.7 \mathrm{GHz}$ Intel Core i7 processor. Each test is for evaluation of 5000 configurations, and the time listed is for evaluating all 5000 configurations

\begin{tabular}{lll}
\hline & Full symmetry & $4-(9,11,11,8)$ \\
\hline Monomials & 52616 & 3609 \\
Polynomials & 6207 & 24030 \\
$N$ (fitted points) & 5454 & 5454 \\
wRMSE (pot) & 49 & 22 \\
wRMSE (grad) & 29 & 16 \\
Time (pot/grad) & $0.95 / 80.91$ & $0.43 / 26.61$
\end{tabular}

however, it must be recalled that the higher symmetry of the full basis results in more complex polynomials, which in this case ends up in significantly increased computational effort. We go back to this table after we discuss the database of energies and gradients.

\subsection{Potential energy surfaces}

The database for the potential energy surface consists of $a b$ initio energies and gradients at 5454 different molecular configurations, using MP2/VTZ level of theory. Of these configurations, 5000 were provided by the authors of ref. 1 , while an additional 454 configurations were generated through grids of points near the transition state to $\mathrm{H}$ transfer and near the global minimum, as well as by running $a b$ initio molecular dynamics trajectories at an energy of $4000 \mathrm{~cm}^{-1}$. A histogram of the distribution of energies is provided in Fig. 2. At these configurations, energies and full gradients were obtained and used in the database. Subsequently, an additional 4 points were added as a result of locating some holes using diffusion Monte Carlo studies (see below). For the $4-(9,11,11,8)$ basis, in order to guarantee that the $\mathrm{H}$-transfer potential is symmetric, the database is expanded by including geometries after the permutation shown in Fig. 3.

Fits using these PIP bases were inverse energy weighted and also gradients were weighted by a factor of $1 / 3$ relative to energies. The precision of these PESs are given in Table 1. As seen the fragmented PES fit is more precise. In particular, the barrier to $\mathrm{H}$ atom transfer, $\mathrm{TS}(\mathrm{H})$ in Table 2 is given more

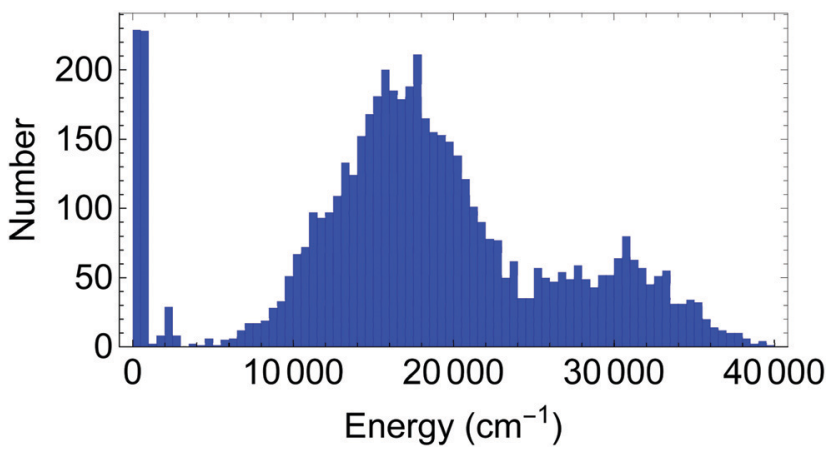

Fig. 2 Histogram of energies for geometries used as the dataset for acetyl-acetone. The bin size for the abscissa is $500 \mathrm{~cm}^{-1}$. The database has 5500 energies and (not shown) $45 \times 5500$ gradient components.

$\begin{array}{llllllll}\mathrm{C}(1) & x_{1} & y_{1} & z_{1} & \mathrm{C}(5) & x_{5} & y_{5} & z_{5} \\ \mathrm{O}(2) & x_{2} & y_{2} & z_{2} & \mathrm{O}(4) & x_{4} & y_{4} & z_{4} \\ \mathrm{H}(3) & x_{3} & y_{3} & z_{3} & \mathrm{H}(3) & x_{3} & y_{3} & z_{3} \\ \mathrm{O}(4) & x_{4} & y_{4} & z_{4} & \mathrm{O}(2) & x_{2} & y_{2} & z_{2} \\ \mathrm{C}(5) & x_{5} & y_{5} & z_{5} & \mathrm{C}(1) & x_{1} & y_{1} & z_{1} \\ \mathrm{C}(6) & x_{6} & y_{6} & z_{6} & \mathrm{C}(12) & x_{12} & y_{12} & z_{12} \\ \mathrm{H}(7) & x_{7} & y_{7} & z_{7} & \mathrm{H}(13) & x_{13} & y_{13} & z_{13} \\ \mathrm{H}(8) & x_{8} & y_{8} & z_{8} & \mathrm{H}(14) & x_{14} & y_{14} & z_{14} \\ \mathrm{H}(9) & x_{9} & y_{9} & z_{9} & \mathrm{H}(15) & x_{15} & y_{15} & z_{15} \\ \mathrm{C}(10) & x_{10} & y_{10} & z_{10} & \mathrm{C}(10) & x_{10} & y_{10} & z_{10} \\ \mathrm{H}(11) & x_{11} & y_{11} & z_{11} & \mathrm{H}(11) & x_{11} & y_{11} & z_{11} \\ \mathrm{C}(12) & x_{12} & y_{12} & z_{12} & \mathrm{C}(6) & x_{6} & y_{6} & z_{6} \\ \mathrm{H}(13) & x_{13} & y_{13} & z_{13} & \mathrm{H}(7) & x_{7} & y_{7} & z_{7} \\ \mathrm{H}(14) & x_{14} & y_{14} & z_{14} & \mathrm{H}(8) & x_{8} & y_{8} & z_{8} \\ \mathrm{H}(15) & x_{15} & y_{15} & z_{15} & \mathrm{H}(9) & x_{9} & y_{9} & z_{9}\end{array}$

Fig. 3 Permutation that must be allowed to describe $\mathrm{H}$-transfer in $\mathrm{Ac}-\mathrm{Ac}$.

Table 2 Energies relative to the GM (in $\mathrm{cm}^{-1}$ ) of the 7 stationary points, and comparison with MP2/aVTZ ones

\begin{tabular}{lccc}
\hline & Full symmetry & $4-(9,11,11,8)$ & MP2/aVTZ \\
\hline GM & 0 & 0 & 0 \\
TS(T)-I & 176 & 159 & 77 \\
TS(T)-II & 270 & 399 & 468 \\
TS(T)-III & 418 & 541 & 519 \\
TS(H) & 714 & 745 & 763 \\
TS(HT)-I & 703 & 820 & 970 \\
TS(HT)-II & 678 & 864 & 1171
\end{tabular}

precisely in the fragmented PES than in the full basis PES. This is important for the predictions of splittings.

\subsection{Diffusion Monte Carlo calculations}

Diffusion Monte Carlo (DMC) simulation is an approach to compute quantum zero-point energy (ZPE) of a molecule, and if the fixed-node approximation is applied, it can also be used to calculate the energies of certain excited states. ${ }^{40,41}$ In this work, we calculate the ground-state splitting of the $\mathrm{H}$-transfer motion by calculating the energies of the ground state and the relevant excited state. This is done for $\mathrm{H}$, a single $\mathrm{D}$ (the transferring atom) and fully deuterated AcAc.

The simple unbiased algorithm, ${ }^{40-42}$ briefly described next, was applied. An ensemble of random walkers is used to represent the nuclear wavefunction of the molecule. At each step, a random displacement in each degree of freedom is assigned to each walker, and this walker may remain alive (and may give birth to a new walker) or be killed by comparing its potential energy, $E_{\mathrm{i}}$, with a reference energy, $E_{\mathrm{r}}$. For the ground state, the probability of birth or death is given as:

$$
\begin{gathered}
P_{\text {birth }}=\exp \left[-\left(E_{\mathrm{i}}-E_{\mathrm{r}}\right) \Delta \tau\right]-1\left(E_{\mathrm{i}}<E_{\mathrm{r}}\right) \\
P_{\text {death }}=1-\exp \left[-\left(E_{\mathrm{i}}-E_{\mathrm{r}}\right) \Delta \tau\right]\left(E_{\mathrm{i}}>E_{\mathrm{r}}\right),
\end{gathered}
$$

where $\Delta \tau$ is the step size in imaginary time. For excited state, in addition to the process described above, any walker that crosses a node is instantly killed. In most cases the node is 
unknown in Cartesian coordinates, but for certain modes such as H-transfer in symmetric wells, a very reasonable approximation can be made for the node as described in detail below.

After removing all dead walkers, the reference energy is updated using the equation

$$
E_{\mathrm{r}}(\tau)=\langle V(\tau)\rangle-\alpha \frac{N(\tau)-N(0)}{N(0)},
$$

where $\tau$ is the imaginary time; $\langle V(\tau)\rangle$ is the average potential over all the walkers that are alive; $N(\tau)$ is the number of live walkers at time $\tau ; \alpha$ is a parameter that can control the fluctuations in the number of walkers and the reference energy. Finally, the average of the reference energy over the imaginary time gives an estimate of ZPE (or the energy of the excited state in a fixed-node calculation).

For AcAc, DMC calculations were performed in two coordinate systems: the Cartesian and normal coordinates. Calculations done in normal coordinates are not rigorous because the Hamiltonian is given as

$$
\hat{H}=-\frac{1}{2} \sum_{k=1}^{3 N-6} \frac{\partial^{2}}{\partial Q_{k}^{2}}+V\left(Q_{1}, \ldots, Q_{3 N-6}\right),
$$

which neglects the vibrational angular momentum terms. The hope is that this error cancels out for tunneling splitting, which is the difference between two energies. The advantage of using normal coordinates is that the node for the fixed-node calculation is much easier to define. The node is just $Q_{\mathrm{im}}=0$, where $Q_{\mathrm{im}}$ is the coordinate of the imaginary-frequency mode of transition state of the $\mathrm{H}$-transfer. That is, whenever the value of $Q_{\text {im }}$ changes sign, the walker should be removed. On the other hand, using Cartesian coordinates is "exact" for the ground state, but more approximations are involved when defining the node for the excited-state calculations. For $\mathrm{H}$ transfer, we assume that the node is where the transferring $\mathrm{H}$ is equidistant from the two $\mathrm{O}$ atoms (i.e., $r_{\mathrm{HO} 2}=r_{\mathrm{HO} 4}$, using the numbering scheme for the $4-(9,11,11,8)$ basis $)$. Initially, the $\mathrm{H}$ atom is closer to one $\mathrm{O}$ (say $\mathrm{O} 4$ ), so if $r_{\mathrm{OH} 4}$ becomes larger than $r_{\mathrm{OH} 2}$ in a walker, that walker crosses the node and should be instantly removed. Also a small correction was made for the excited state by taking recrossing into consideration. ${ }^{41}$

Ten DMC simulations were performed for each state and each coordinate system, and for three deuterated isotopologues (all $\mathrm{H}$, all $\mathrm{D}$, and one $\mathrm{D}$ ). so the total number of simulations is $10 \times 2 \times 2 \times 3=120$. In each simulation, 30000 walkers were equilibrated for 5000 steps, and then were propagated for 50000 steps to compute the energy, with a step size of 5.0 a.u. In these simulations, $\sim 10^{11}$ potential energy evaluations are required; clearly these cannot be done without an efficient PES.

\section{Results and discussion}

\subsection{Potential energy surface}

Seven low-energy stationary points can be located on the PES: the global minimum (GM), 3 transition states with respect to the torsion of the two methyl rotors (denoted as TS(T)-I, TS(T)-II, and TS(T)-III), the transition state of the $\mathrm{H}$ transfer (denoted as $\mathrm{TS}(\mathrm{H})$ ), and 2 higher-order saddle points with imaginary frequencies in both H-transfer motion and the methyl torsion (denoted as TS(HT)-I and TS(HT)-II). The geometries of GM and TS(H) are shown in Fig. 4, and the coordinates and harmonic frequencies of all the 7 stationary points can be found in the ESI. $\uparrow$ The GM has $C_{\mathrm{s}}$ symmetry, while the H-transfer transition state is a $C_{2 \mathrm{v}}$ structure.

The energies of the 7 stationary points from two PESs are shown in Table 2, and compared with MP2/aVTZ values. It can be seen that the $4-(9,11,11,8)$ PES has better agreement with MP2 than the PES using full symmetry basis, as expected, due to the smaller fitting errors shown in Table 1 . The $4-(9,11,11,8)$ PES also shows better agreement with MP2 harmonic frequencies; the mean absolute errors (MAE) in frequencies are listed in Table 3 .

There are two methyl rotors in AcAc; by allowing the permutations of the three $\mathrm{H}$ atoms in each rotor, the fragmented $4-(9,11,11,8)$ can still ensure the 3 -fold symmetry of the methyl rotors, as is shown in Fig. 5 .

From data reported in Table 1 it is possible to estimate the computational cost of a single AcAc energy evaluation performed on a $2.7 \mathrm{GHz}$ Intel Core i7 processor. We found the 4-fragment surface to be faster than the full symmetry one. The result for the 4 -fragment surface is about $0.08 \mathrm{~ms}$ per energy, while one gradient call is about 55 times more expensive at about $5 \mathrm{~ms}$. This is to be compared with values reported in ref. 1 for an NN PES of the smaller malonaldehyde molecule.
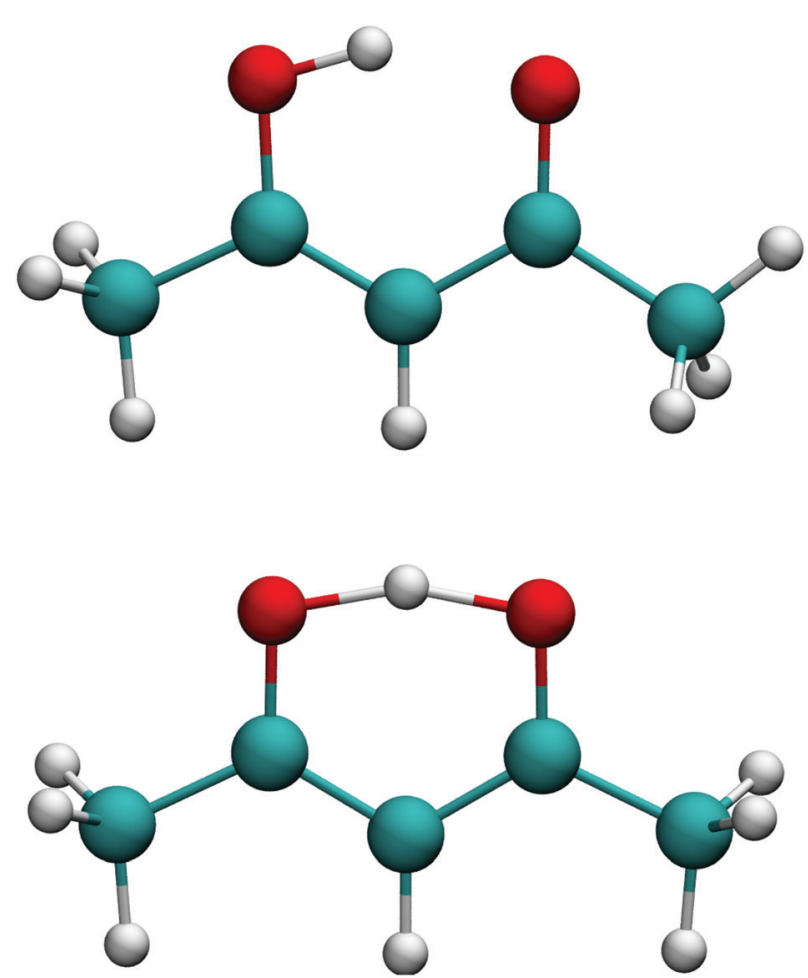

Fig. 4 Geometries of GM (top) and H-transfer saddle point (bottom). 
Table 3 Mean absolute error (MAE) in harmonic frequencies compared to those computed at MP2/aVTZ level of theory. All values are in $\mathrm{cm}^{-1}$

\begin{tabular}{lll}
\hline & Full symmetry & $4-(9,11,11,8)$ \\
\hline GM & 22.2 & 14.4 \\
TS(T)-I & 19.0 & 13.2 \\
TS(T)-II & 28.2 & 18.1 \\
TS(T)-III & 24.0 & 15.7 \\
TS(H) & 31.8 & 20.4 \\
TS(HT)-I & 31.0 & 21.5 \\
TS(HT)-II & 38.2 & 23.5
\end{tabular}

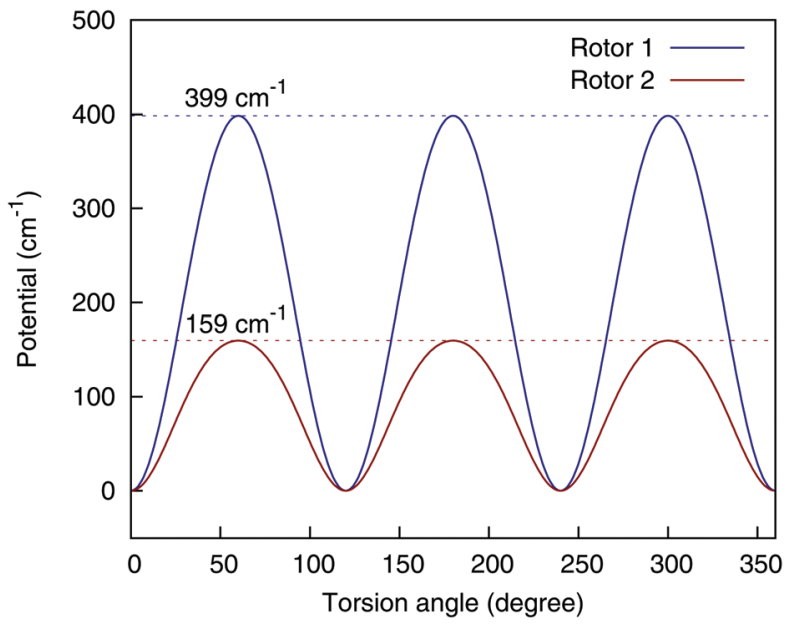

Fig. 5 Torsional potential of the two methyl rotors, using the $4-(9,11,11,8)$ basis. Rotor 1 consists of atom $\{6,7,8,9\}$, and rotor 2 consists of atom $\{12,13,14,15\}$, using the numbering scheme in the bottom panel of Fig. 1

In that paper one potential and one gradient evaluation on a faster $(3.4 \mathrm{GHz})$ processor were timed at 4 and $9 \mathrm{~ms}$ respectively. This fact hints at substantially increased efficiency of our fragmented PIP PES. However, we cannot rule out that part of the PIP speedup might be due to the way the surface was coded (Fortran 90 for the PIP PES; Python for the NN malonaldehyde PES) rather than the way it was built. Overall, then, our PES is full-dimensional and does describe satisfactorily the region of the stationary points shown.

\subsection{Splitting dynamics}

All DMC and 1d calculations are done with the fragmented PES, which is both faster and more precise than the full basis PES. Before discussing the tunneling splitting dynamics, we present the ZPEs of three isotopologues of AcAc and the groundstate wavefunction, which are also important properties and closely related to tunneling. The ZPEs of un-deuterated, singly deuterated, and fully deuterated AcAc from DMC calculations in Cartesian coordinates are $26637 \pm 16 \mathrm{~cm}^{-1}, 26048 \pm$ $7 \mathrm{~cm}^{-1}$, and $21223 \pm 12 \mathrm{~cm}^{-1}$, respectively. The numbers from DMC in normal coordinates are $26587 \pm 6 \mathrm{~cm}^{-1}, 26005 \pm$ $8 \mathrm{~cm}^{-1}$, and $21177 \pm 16 \mathrm{~cm}^{-1}$; the difference between Cartesian and normal coordinates, roughly $45 \mathrm{~cm}^{-1}$, is due to the neglect of vibrational angular momentum terms when using normal coordinates.

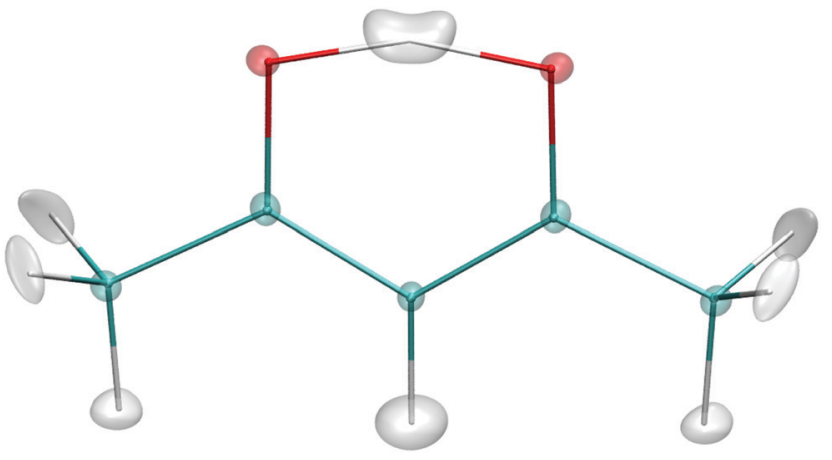

Fig. 6 Isosurface depiction of the ground vibrational state wavefunction.

Fig. 6 shows the isosurface depiction of the ground vibrational state wavefunction. As seen the "structure" is close to the $\mathrm{H}$-transfer saddle point with an effective symmetry of $C_{2 \mathrm{v}}$.

The tunneling splittings of $\mathrm{H}$-atom and two deuterated isotopologues obtained from DMC calculations and a 1d approach (described below) are summarized in Table 4. As seen, the DMC results using Cartesian coordinates and normal coordinates are in good agreement and this indicates a small systematic uncertainty in using these different approaches. It should be noted that statistical uncertainties for each set of DMC calculations are larger than these differences. Also, we stress that these DMC splittings are not expected to be absolutely accurate owing to the MP2/aVTZ underestimation of the $\mathrm{H}$-atom transfer barrier height by roughly $1 \mathrm{kcal} \mathrm{mol}^{-1}$. At the suggestion of a Reviewer we investigated using a CBS extrapolation based on MP2/aVDZ and MP2/aVTZ energies. ${ }^{43}$ We found a $0.1 \mathrm{kcal} \mathrm{mol}^{-1}$ lowering of the H-transfer barrier height doing this. This is a small change and actually in the direction away from the higher $\operatorname{CCSD}(\mathrm{T})$ barrier, so we did not pursue this further.

In view of this, we investigated the dependency of the splitting as a function of the barrier height, using a simple $1 \mathrm{~d}$ approach. In brief, a $1 \mathrm{~d}$ potential, denoted $V\left(Q_{\mathrm{im}}\right)$, which is the minimum energy path as a function of the imaginaryfrequency mode $\left(Q_{\mathrm{im}}\right)$ of the H-transfer saddle point, was obtained by optimizing all the other coordinates at fixed $Q_{\mathrm{im}}$ values except the methyl rotors, which cannot be described using rectilinear normal coordinates. These are held fixed at the saddle point values all the way along the path. Then 1d-DVR calculations were done using the $1 \mathrm{~d}$ potential to compute the energies of the ground and first excited states, and thus the splitting.

Table 4 Ground-state tunneling splittings of three deuterated isotopologues of AcAc obtained by the specified approaches. The numbers in parenthesis indicate the barrier height

\begin{tabular}{llll}
\hline & All $\mathrm{H}$ & One D & All D \\
\hline DMC (Cartesian) & 160 & 43 & 37 \\
DMC (normal) & 156 & 40 & 36 \\
1d-DVR $\left(586 \mathrm{~cm}^{-1}\right)$ & 141 & 57 & 57 \\
1d-DVR $\left(763 \mathrm{~cm}^{-1}\right)$ & 113 & 41 & 40 \\
1d-DVR $\left(1119 \mathrm{~cm}^{-1}\right)$ & 74 & 22 & 21
\end{tabular}


Owing to fixing the methyl rotors the $1 \mathrm{~d} Q_{\text {im }}$ potential has a barrier to $\mathrm{H}$ or $\mathrm{D}$ transfer of $586 \mathrm{~cm}^{-1}$ relative to the minima at $Q_{\min }= \pm 27.9$ a.u. for $\mathrm{H}$ transfer and $Q_{\min }= \pm 36.8$ a.u. for $\mathrm{D}$ transfer. Thus, The $1 \mathrm{~d} Q_{\text {im }}$ barrier is about $160 \mathrm{~cm}^{-1}$ less than the barriers of $745 \mathrm{~cm}^{-1}$ for the fitted surface or $763 \mathrm{~cm}^{-1}$ for the MP2 result. The 1d-DVR splittings for $\mathrm{H}$ and $\mathrm{D}$ transfer were found to be $141 \mathrm{~cm}^{-1}$ and $57 \mathrm{~cm}^{-1}$, respectively for this barrier height of $586 \mathrm{~cm}^{-1}$.

In order to investigate the dependence of the splitting on the barrier height a simple potential morphing approach was used. ${ }^{44-46}$ Specifically, the morphed $1 \mathrm{~d}$ potential is $\tilde{V}\left(Q_{\mathrm{im}}\right)=$ $f\left(Q_{\text {im }}\right) V\left(Q_{\text {im }}\right)$, where $V\left(Q_{\text {im }}\right)$ is the potential from the original PES, and $f\left(Q_{\mathrm{im}}\right)$ is the scaling factor, which is 1.0 at the minima $\left(Q_{\mathrm{im}}=Q_{\mathrm{min}}\right)$ and equal to a value greater than 1.0 that can bring the barrier to the desired height at $Q_{\mathrm{im}}=0.0$. The scaling factor we employed is

$$
f\left(Q_{\mathrm{im}}\right)=\left(R_{\mathrm{bh}}-1\right)\left[1-s\left(Q_{\mathrm{im}}\right)\right]+1,
$$

where $R_{\mathrm{bh}}$ is the ratio between the desired barrier height plus $159 \mathrm{~cm}^{-1}$ and the actual barrier height from the PES (which is $745 \mathrm{~cm}^{-1}$ ), and $s$ is a switching function that is 0 at $Q_{\mathrm{im}}=0$ and is 1 at $Q_{\text {im }}=Q_{\text {min }}$. Note that adding $159 \mathrm{~cm}^{-1}$ to the desired barrier height is due to the fact that the minimum in the $1 \mathrm{~d} Q_{\mathrm{im}}$ potential has an energy of $159 \mathrm{~cm}^{-1}$ instead of 0 . The switching function has the form

$$
s=10\left(\frac{Q_{\mathrm{im}}}{Q_{\mathrm{min}}}\right)^{3}-15\left(\frac{Q_{\mathrm{im}}}{Q_{\mathrm{min}}}\right)^{4}+6\left(\frac{Q_{\mathrm{im}}}{Q_{\mathrm{min}}}\right)^{5} .
$$

The results from various morphed potentials are summarized in Table 4. As seen and as expected, the splittings decrease significantly in going from the barrier height of $763 \mathrm{~cm}^{-1}$ to $1119 \mathrm{~cm}^{-1}$.

The simple morphing of the $1 \mathrm{~d} Q_{\text {im }}$ potentials would not be expected to work for the PES in full dimensionality. (Indeed the attempt to do so was not satisfactory.) A multi-dimensional approach is probably needed and we present some preliminary thoughts on this in the next section.

To summarize this section, we finally use the 1d-DVR splitting results in Table 4 to estimate the DMC splittings corresponding to the barrier of $1119 \mathrm{~cm}^{-1}$. This is done just using the ratio of the $1 \mathrm{~d}$ splitting for 763 and $1119 \mathrm{~cm}^{-1}$. From these we estimate $104 \mathrm{~cm}^{-1}$ for $\mathrm{H}$-atom transfer and 23 and $19 \mathrm{~cm}^{-1}$ for the single-D and all-D isotopologues, respectively.

\section{Morphing with Gaussian process regression}

Morphing is a term that refers to a procedure to change an existing PES with various goals in mind. In the simplest version, a coordinate is scaled by a dimensionless factor to bring "exact" numerical vibrational transition energies into agreement with experiment. $^{44}$ A more elaborate procedure was applied to an approximate semi-empirical PES describing HCN/HNC isomerization to change the isomerization saddle point location as well as to increase the energy substantially. ${ }^{45}$ Meuwly and Hutson used the general approach of scaling a coordinate and the PES energy to obtain a highly accurate intermolecular PES for Ne-HF. ${ }^{46}$

In these approaches the scaling was done using analytical expressions. This could be done without difficulty for these triatomics since there are only three internal degrees of freedom. For larger molecules with tens of degrees of freedom a more general, non-parametric approach is probably called for. This is essentially the suggestion made in this short section, motivated by the present PES for AcAc.

As noted both here and previously ${ }^{1}$ the $\operatorname{CCSD}(\mathrm{T})$ barrier height for $\mathrm{H}$-atom transfer is roughly $50 \%$ higher than the MP2 one. Thus, even a perfect fit to MP2 energies would produce an inaccurate barrier. Morphing such a PES to produce the correct $\operatorname{CCSD}(\mathrm{T})$ barrier obviously would be the goal. However, in principle a scaling factor to do this would be function of all the variables of the PES. In addition, moving the configuration of the saddle point to the "correct" one would require scaling of all the variables, which in the present case are all the Morse variables. We note that an approach based on energy switching to build single and multi-sheeted PESs to account for improved barrier descriptions has been developed by Varandas and co-workers. ${ }^{47}$

To improve the MP2-based AcAc PES Meuwly and co-workers proposed a transfer learning approach based on approximate local $\operatorname{CCSD}(\mathrm{T})$ energies (no gradients) at 49000 geometries for the series malonaldehyde, acetoacetaldehyde, and acetylacetone. This appeared to be successful, however 49000 full CCSD(T) energies just for AcAc would be prohibitive. Our proposal is to make use of on the order of a thousand $\operatorname{CCSD}(\mathrm{T})$ energies strategically done, at least at and in the vicinity of stationary points. Then an efficient ML approach could be applied to develop high dimensional morphing functions. Gaussian process regression appears to be an excellent choice as it is known to perform well on relative small data sets. ${ }^{11,48}$ We plan to do this in the near future.

\section{Summary and conclusions}

We reported permutationally invariant polynomial (PIP) fits to energies and gradient components for 15-atom acetylacetone. These include standard and fragmented PIP bases. Approximately 500 energies and associated gradient components are obtained from direct-dynamics calculations using MP2/avTZ supplemented by grid calculations spanning an energy range up to roughly $35000 \mathrm{~cm}^{-1}$, and are combined with an existing database $^{1}$ to form the database for this study. A fragmentation scheme was investigated with respect to efficiency and fit precision. Properties such as stationary points, harmonic frequencies and the barrier to $\mathrm{H}$-atom transfer are reported and compared to direct calculations. Diffusion Monte Carlo (DMC) calculations are used to calculate the ground state wavefunction and zero-point energy of acetylacetone. These together with fixed-node DMC calculations for the first excited-state of $\mathrm{H}$-transfer provide the predicted tunneling splitting due to the barrier separating two equivalent wells. Simpler $1 \mathrm{~d}$ calculations 
of this splitting are also reported for varying barrier heights including the $\operatorname{CCSD}(\mathrm{T})$ barrier height of $3.2 \mathrm{kcal} \mathrm{mol}^{-1}$. Based on those results the DMC splitting of roughly $160 \mathrm{~cm}^{-1}$ with a statistical uncertainty of about $30 \mathrm{~cm}^{-1}$, calculated using the MP2-based PES, is estimated to diminish to about $100 \mathrm{~cm}^{-1}$ for a barrier of $3.2 \mathrm{kcal} \mathrm{mol}^{-1}$.

In the abstract and along the text we have adopted the expressions "full basis" or "full symmetry" to refer to the non-fragmented fit. This is to mean that a unique fitting basis extended to the full molecule has been employed with the theoretical possibility for all atoms to permute with same-kind ones. In practice we generated a basis set characterized by partial permutational symmetry to be able to deal with the large dimensionality of the molecule (15 atoms), as detailed in Section 3.1. This is expected since fully permutationally invariant bases have generally been limited to 10 -atom systems.

We conclude spending a few words about the possibility to employ fragmentation to reactive or dissociative systems, as already done in one of our previous papers. ${ }^{19}$ It should be possible to reduce the basis set of a dissociating molecule by choosing fragments that either (a) remain in one of the products or (b) are the simplest fragments that represent the dissociation. In the end, of course, the choice of basis polynomials and the $a b$ initio data set are closely linked. If the data set is chosen, it is easy to examine it to see which Morse variables are most and least important. But if the data set is subsequently extended, say to examine a particular conformer or product, the choice of the most or least important Morse variables must be re-evaluated. Preliminary and encouraging work in this direction is being undertaken.

Finally, the fragmented PES is available upon request to the authors.

\section{Conflicts of interest}

There are no conflicts to declare.

\section{Acknowledgements}

We thank Silvan Käser and Markus Meuwly for sending their MP2 dataset and for help using it. JMB thanks the Army Research Office, DURIP grant (W911NF-14-1-0471), for funding a computer cluster where most of the calculations were performed.

\section{Notes and references}

1 S. Käser, O. Unke and M. Meuwly, New J. Phys., 2020, 22, 055002.

2 B. J. Braams and J. M. Bowman, Int. Rev. Phys. Chem., 2009, 28, 577-606.

3 J. Cui and R. V. Krems, J. Phys. B: At., Mol. Opt. Phys., 2016, 49, 224001.

4 A. P. Bartók and G. Csányi, Int. J. Quantum Chem., 2015, 115, 1051-1057.

5 J. Behler, Int. J. Quantum Chem., 2015, 115, 1032-1050.
6 B. Jiang, J. Li and H. Guo, Int. Rev. Phys. Chem., 2016, 35, 479-506.

7 C. Qu, Q. Yu and J. M. Bowman, Annu. Rev. Phys. Chem., 2018, 69, 6.1-6.25.

8 B. Fu and D. H. Zhang, J. Chem. Theory Comput., 2018, 14, 2289-2303.

9 B. Jiang, J. Li and H. Guo, J. Phys. Chem. Lett., 2020, 11, 5120-5131.

10 T. T. Nguyen, E. Székely, G. Imbalzano, J. Behler, G. Csányi, M. Ceriotti, A. W. Götz and F. Paesani, J. Chem. Phys., 2018, 148, 241725.

11 C. Qu, Q. Yu, B. L. Van Hoozen, J. M. Bowman and R. A. Vargas-Hernández, J. Chem. Theory Comput., 2018, 14, 3381-3396.

12 J. Li, K. Song and J. Behler, Phys. Chem. Chem. Phys., 2019, 21, 9672-9682.

13 D. Lu, J. Behler and J. Li, J. Phys. Chem. A, 2020, 124, 5737-5745.

14 K. Shao, J. Chen, Z. Zhao and D. H. Zhang, J. Chem. Phys., 2016, 145, 071101.

15 J. Dai and R. V. Krems, J. Chem. Theory Comput., 2020, 16, 1386-1395.

16 R. C. Fortenberry, Q. Yu, J. S. Mancini, J. M. Bowman, T. J. Lee, T. D. Crawford, W. F. Klemperer and J. S. Francisco, J. Chem. Phys., 2015, 143, 071102.

17 A. Nandi, C. Qu and J. M. Bowman, J. Chem. Theory Comput., 2019, 15, 2826-2835.

18 A. Nandi, C. Qu and J. M. Bowman, J. Chem. Phys., 2019, 151, 084306.

19 P. L. Houston, R. Conte, C. Qu and J. M. Bowman, J. Chem. Phys., 2020, 153, 024107.

20 J. M. Bowman, B. J. Braams, S. Carter, C. Chen, G. Czako, B. Fu, X. Huang, E. Kamarchik, A. R. Sharma, B. C. Shepler, Y. Wang and Z. Xie, J. Phys. Chem. Lett., 2010, 1, 1866-1874.

21 J. M. Bowman, G. Czakó and B. Fu, Phys. Chem. Chem. Phys., 2011, 13, 8094-8111.

22 J. Mavri and J. Grdadolnik, J. Phys. Chem. A, 2001, 105, 2039-2044.

23 B. Fu, Y.-C. Han, J. M. Bowman, L. Angelucci, N. Balucani, F. Leonori and P. Casavecchia, Proc. Natl. Acad. Sci. U. S. A., 2012, 109, 9733-9738.

24 Z. Xie and J. M. Bowman, J. Chem. Theory Comput., 2010, 6, 26-34.

25 B. J. Braams and J. M. Bowman, Int. Rev. Phys. Chem., 2009, 28, 577-606.

26 R. Chen, K. Shao, B. Fu and D. H. Zhang, J. Chem. Phys., 2020, 152, 204307.

27 Y. Wang, B. Braams, J. M. Bowman, S. Carter and D. P. Tew, J. Chem. Phys., 2008, 128, 224314.

28 C. Qu and J. M. Bowman, J. Chem. Phys., 2019, 150, 141101.

29 R. Conte, P. L. Houston and J. M. Bowman, J. Chem. Phys., 2014, 140, 151101.

30 R. Conte, C. Qu and J. M. Bowman, J. Chem. Theory Comput., 2015, 11, 1631-1638.

31 C. Qu, R. Conte, P. L. Houston and J. M. Bowman, Phys. Chem. Chem. Phys., 2015, 17, 8172-8181. 
32 K. Hinsen and B. Roux, J. Chem. Phys., 1997, 106, 3567-3577.

33 K. Hinsen and B. Roux, J. Comput. Chem., 1997, 18, 368-380.

34 D. L. Howard, H. G. Kjaergaard, J. Huang and M. Meuwly, J. Phys. Chem. A, 2015, 119, 7980-7990.

35 O. T. Unke and M. Meuwly, J. Chem. Theory Comput., 2019, 15, 3678-3693.

36 Z. Jin, B. J. Braams and J. M. Bowman, J. Phys. Chem. A, 2006, 110, 1569-1574.

37 X. Huang, B. J. Braams and J. M. Bowman, J. Chem. Phys., 2005, 122, 044308.

38 A. Nandi, C. Qu and J. M. Bowman, J. Chem. Theory Comput., 2019, 15, 2826-2835.

39 R. Conte, C. Qu, P. L. Houston and J. M. Bowman, J. Chem. Theory Comput., 2020, 16, 3264-3272.

40 J. B. Anderson, J. Chem. Phys., 1975, 63, 1499-1503.
41 J. B. Anderson, J. Chem. Phys., 1976, 65, 4121-4127.

42 I. Kosztin, B. Faber and K. Schulten, Am. J. Phys., 1996, 64, 633-644.

43 H.-J. Werner, P. J. Knowles, G. Knizia, F. R. Manby and M. Schütz, MOLPRO, version 2015.1, a package of ab initio programs, 2015, see http://www.molpro.net.

44 J. M. Bowman and B. Gazdy, J. Chem. Phys., 1991, 94, 816-817. 45 B. Gazdy and J. M. Bowman, J. Chem. Phys., 1991, 95, 6309-6316.

46 M. Meuwly and J. M. Hutson, J. Chem. Phys., 1999, 110, 8338-8347.

47 B. R. L. Galvao, S. P. J. Rodrigues and A. J. C. Varandas, J. Chem. Phys., 2008, 129, 044302.

48 A. Kamath, R. A. Vargas-Hernández, R. Krems, T. Carrington and S. Manzhos, J. Chem. Phys., 2018, 148, 241702. 